TTP95-32

hep-ph/9509204

August 1995

\title{
Strategies for Fixing the CKM-angle $\gamma$ and Obtaining Experimental Insights into the World of Electroweak Penguins
}

\author{
ROBERT FLEISCHER \\ Institut für Theoretische Teilchenphysik \\ Universität Karlsruhe \\ D-76128 Karlsruhe, Germany
}

\begin{abstract}
Using the $S U(3)$ flavour symmetry of strong interactions, we propose strategies for extracting both the CKM-angle $\gamma$ and the $\bar{b} \rightarrow \bar{u} u \bar{s}$ tree-level amplitude $T^{\prime}$. We present also an approximate approach using the branching ratios for the modes $B^{+} \rightarrow \pi^{+} K^{0}$, $B_{d}^{0} \rightarrow \pi^{-} K^{+}, \bar{B}_{d}^{0} \rightarrow \pi^{+} K^{-}$and $B^{+} \rightarrow \pi^{+} \pi^{0}$ which should be rather promising from the experimental point of view. The quantities $\gamma$ and $T^{\prime}$ determined this way may well be used as an input to control electroweak penguins in nonleptonic $B$-decays as has been discussed in previous work. Following these lines, we propose strategies for obtaining quantitative insights into the physics of the electroweak penguin operators and performing some consistency checks. As a by-product, we derive an upper bound of $6^{\circ}$ for the uncertainty originating from electroweak penguins in the $\alpha$-determination by means of $B \rightarrow \pi \pi$ decays.
\end{abstract}



Strategies for the determination of the angle $\gamma$ in the unitarity triangle [1, 2] are among the central issues of present particle physics phenomenology. Although there are already methods on the market allowing an absolutely clean measurement of this quantity (see e.g. refs. [3]-[6]), they are quite challenging for experimentalists. An interesting approach to measure both weak and strong phases by using $S U(3)$ triangle relations among $B \rightarrow\{\pi \pi, \pi K, K \bar{K}\}$ decays and making some plausible dynamical assumptions (neglect of annihilation topologies, etc.) was proposed last year by Gronau, Hernández, London and Rosner [7]-[12].

Unfortunately, similar to the situation arising in certain nonleptonic $B$-meson decays [13-18, electroweak penguins may have a considerable impact on this approach and may in particular preclude a clean determination of the CKM-angle $\gamma$ [19, 20]. In order to eliminate the electroweak penguin contributions, Gronau et al. have constructed an amplitude quadrangle involving $B \rightarrow \pi K$ decays [20] that can be used in principle to extract $\gamma$. However, this approach is very difficult from the experimental point of view, since one diagonal of the quadrangle corresponds to the decay $B_{s} \rightarrow \pi^{0} \eta$ which is expected to have a very small branching ratio at the $\mathcal{O}\left(10^{-7}\right)$ level. Recently, Deshpande and He have presented another $S U(3)$-based method [21] which uses the charged $B$-decays $B^{-} \rightarrow\left\{\pi^{-} \bar{K}^{0}, \pi^{0} K^{-}, \eta K^{-}, \pi^{-} \pi^{0}\right\}$ and is unaffected by electroweak penguins as well. Although this approach is more promising for experimentalists the relevant branching ratios are $\mathcal{O}\left(10^{-5}\right)$ - it suffers from $\eta-\eta^{\prime}$-mixing and other $S U(3)$-breaking effects.

In a recent publication [22], it has been shown that the $\bar{b} \rightarrow \bar{s}$ electroweak penguin amplitude $\left(c_{u}-c_{d}\right) P_{\mathrm{EW}}^{\prime}$ can be determined from $B \rightarrow \pi K$ decays having branching ratios $\mathcal{O}\left(10^{-5}\right)$ if one uses the CKM-angle $\gamma$ as one of the central inputs and makes some reasonable approximations. Since electroweak penguins are - in contrast to QCD penguins 23] - dominated to a good approximation by internal top-quark exchanges, the $\bar{b} \rightarrow \bar{s}$ electroweak penguin amplitude can be related to the $\bar{b} \rightarrow \bar{d}$ amplitude $\left(c_{u}-c_{d}\right) P_{\text {EW }}$ by using the $S U(3)$ flavour symmetry of strong interactions. The knowledge of $\left(c_{u}-c_{d}\right) P_{\mathrm{EW}}$ allows in particular to investigate whether the uncertainty $\Delta \alpha$ in the Gronau-London-method [24] of measuring the CKM-angle $\alpha$ introduced by electroweak penguin effects is really as small as is expected from theoretical estimates [19, 20]. As we shall see below, an upper limit for this uncertainty is given by $|\Delta \alpha| \lesssim 6^{\circ}$.

In this letter we would like to discuss some other applications of the approach 22] which allow quantitative insights into the physics of the electroweak penguin operators and provide interesting tests of the Standard Model of electroweak interactions [25]. As the CKM-angle $\gamma$ is one of the central ingredients of this method, let us begin our discussion by presenting a new strategy for extracting this quantity. To this end we consider the decays $B^{+} \rightarrow \pi^{+} K^{0}$ and $B_{d}^{0} \rightarrow \pi^{-} K^{+}$. If we apply the same nota- 
tion as Gronau, Hernández, London and Rosner in ref. [20], the corresponding decay amplitudes take the form

$$
\begin{aligned}
& A\left(B^{+} \rightarrow \pi^{+} K^{0}\right)=P^{\prime}+c_{d} P_{\mathrm{EW}}^{\prime \mathrm{C}} \\
& A\left(B_{d}^{0} \rightarrow \pi^{-} K^{+}\right)=-\left(P^{\prime}+T^{\prime}+c_{u} P_{\mathrm{EW}}^{\prime \mathrm{C}}\right)
\end{aligned}
$$

where $T^{\prime}$ describes the colour-allowed $\bar{b} \rightarrow \bar{u} u \bar{s}$ tree-level amplitude, $P^{\prime}$ denotes $\bar{b} \rightarrow \bar{s}$ QCD penguins and $P_{\mathrm{EW}}^{\prime \mathrm{C}}$ is related to colour-suppressed electroweak penguins. Neglecting as in [22] the colour-suppressed electroweak penguin contributions, we obtain

$$
\begin{aligned}
& A\left(B^{+} \rightarrow \pi^{+} K^{0}\right)=P^{\prime}=A\left(B^{-} \rightarrow \pi^{-} \bar{K}^{0}\right) \\
& A\left(B_{d}^{0} \rightarrow \pi^{-} K^{+}\right)=-\left(P^{\prime}+T^{\prime}\right) \\
& A\left(\bar{B}_{d}^{0} \rightarrow \pi^{+} K^{-}\right)=-\left(P^{\prime}+e^{-2 i \gamma} T^{\prime}\right),
\end{aligned}
$$

where we have taken into account the relations

$$
\begin{gathered}
P^{\prime}=\left|P^{\prime}\right| e^{i \delta_{P^{\prime}}} e^{i \pi}=\bar{P}^{\prime} \\
\bar{T}^{\prime}=e^{-2 i \gamma} T^{\prime} .
\end{gathered}
$$

Note that (3) arises from the special CKM-structure of the $\bar{b} \rightarrow \bar{s}$ penguins [23] and that $\delta_{P^{\prime}}$ and $\pi$ are CP-conserving strong and CP-violating weak phases, respectively. In Fig. 1 we have represented (2) graphically in the complex plane. Looking at this figure, which is a modification of Fig. 2 given in ref. [22], implies that both the quantities $z \equiv T^{\prime} /\left|P^{\prime}\right|, \bar{z} \equiv \bar{T}^{\prime} /\left|P^{\prime}\right|$ and the CKM-angle $\gamma$ can be determined from the measured branching ratios $\mathrm{BR}\left(B^{+} \rightarrow \pi^{+} K^{0}\right)=\operatorname{BR}\left(B^{-} \rightarrow \pi^{-} \bar{K}^{0}\right), \operatorname{BR}\left(B_{d}^{0} \rightarrow \pi^{-} K^{+}\right)$and $\operatorname{BR}\left(\bar{B}_{d}^{0} \rightarrow \pi^{+} K^{-}\right)$, if $\left|T^{\prime}\right|=\left|\bar{T}^{\prime}\right|$ is known. This quantity can be determined by relating it to the $\bar{b} \rightarrow \bar{u} u \bar{d}$ colour-allowed tree-level amplitude $T$. Making use of the $S U(3)$ flavour symmetry of strong interactions, one finds [12]

$$
\frac{\left|T^{\prime}\right|}{|T|}=\lambda \frac{f_{K}}{f_{\pi}},
$$

where $\lambda=0.22$ is the usual Wolfenstein parameter [26] and $f_{K}$ and $f_{\pi}$ are the $K$ - and $\pi$-meson decay constants, respectively, describing factorizable $S U(3)$-breaking. Unfortunately, non-factorizable $S U(3)$-breaking corrections to (5) are completely unknown at present and therefore cannot be included. In view of these uncertainties, the neglect of the colour-suppressed electroweak penguins in (2), which are expected to be suppressed by factors $\mathcal{O}\left(\bar{\lambda}^{2}\right)$ and $\mathcal{O}(\bar{\lambda})$ relative to the amplitudes $P^{\prime}$ and $T^{\prime}$, respectively, seems to be reasonable. The parameter $\bar{\lambda}=\mathcal{O}(0.2)$ describes the hierarchy of the different topologies contributing to $B \rightarrow P P$ decays [12, 20, 22].

The quantity $|T|$ can be extracted in principle in a clean way up to corrections of $\mathcal{O}\left(\bar{\lambda}^{2}\right)$ by applying the approach presented in [27]. There, a determination of the 
CKM-angle $\alpha$ by using mixing-induced CP violation in the decays $B_{d} \rightarrow \pi^{+} \pi^{-}$and $B_{d} \rightarrow K^{0} \bar{K}^{0}$ has been proposed. As a by-product of this analysis, the quantity $|T+E|$, where $E$ corresponds to an $\mathcal{O}\left(\bar{\lambda}^{2}\right)$-suppressed exchange amplitude, is also fixed and can be used to determine $\left|T^{\prime}\right|$ through (5). This approach is, however, quite difficult for experimentalists. Therefore, it is an important question to search also for methods of obtaining approximate information on $|T|$ that can be realized more easily in practice.

Such an estimate of $|T|$ can be obtained directly from the branching ratio for the decay $B^{+} \rightarrow \pi^{+} \pi^{0}$. In the notation of ref. [20] its transition amplitude takes the form

$$
A\left(B^{+} \rightarrow \pi^{+} \pi^{0}\right)=-\frac{1}{\sqrt{2}}\left[T+C+\left(c_{u}-c_{d}\right)\left(P_{\mathrm{EW}}+P_{\mathrm{EW}}^{\mathrm{C}}\right)\right]
$$

If we neglect both the colour-suppressed $\bar{b} \rightarrow \bar{u} u \bar{d}$ tree-level amplitude $C$ and the very small electroweak penguin contributions, which should be suppressed relative to $T$ by factors $\mathcal{O}(\bar{\lambda})$ and $\mathcal{O}\left(\bar{\lambda}^{2}\right)$, respectively [20], we find

$$
|T| \approx \sqrt{2}\left|A\left(B^{+} \rightarrow \pi^{+} \pi^{0}\right)\right|
$$

This approximation is consistent with those performed in [22] where the coloursuppressed $\bar{b} \rightarrow \bar{u} u \bar{s}$ tree-level amplitude $\left|C^{\prime}\right|$ has also been neglected. Consequently, combining (5) and (7), the amplitudes $z$ and $\bar{z}$, which are essential for the determination of the $\bar{b} \rightarrow \bar{s}$ electroweak penguin amplitude $\left(c_{u}-c_{d}\right) P_{\mathrm{EW}}^{\prime}$ as has been outlined in [22], can be extracted from Fig. 1. Since the angle between these amplitudes is given by $2 \gamma$ it can be fixed as well. This determination of $\gamma$ is just an estimate and cannot be considered as a precision measurement. However, it should be rather promising from the experimental point of view because all involved branching ratios are $\mathcal{O}\left(10^{-5}\right)$. Moreover, it requires a quite simple geometrical construction, which is very similar to the original one suggested by Gronau, Rosner and London [7], and is not affected by $\eta-\eta^{\prime}$-mixing as the method for determining $\gamma$ proposed by Deshpande and He 21.

The quantities $z$ and $\bar{z}$ may well be used to obtain information on $\bar{b} \rightarrow \bar{s}$ electroweak penguins by following the approach presented in ref. [22]. In this paper, different strategies for determining $z$ and $\bar{z}$ that can be used if the CKM-angle $\gamma$ is known, for example by applying the absolutely clean method of Gronau and Wyler [3], have also been discussed.

The scenario at future experimental $B$-physics facilities might be as follows:

- In the $1^{\text {st }}$ generation of experiments it should be possible to estimate $z, \bar{z}$ and $\gamma$ by measuring the four branching ratios $\mathrm{BR}\left(B^{+} \rightarrow \pi^{+} K^{0}\right)=\mathrm{BR}\left(B^{-} \rightarrow \pi^{-} \bar{K}^{0}\right)$, $\mathrm{BR}\left(B_{d}^{0} \rightarrow \pi^{-} K^{+}\right), \mathrm{BR}\left(\bar{B}_{d}^{0} \rightarrow \pi^{+} K^{-}\right)$and $\mathrm{BR}\left(B^{+} \rightarrow \pi^{+} \pi^{0}\right)$ as we have proposed above. If one measures in addition the two branching ratios $\operatorname{BR}\left(B^{+} \rightarrow \pi^{0} K^{+}\right)$ and $\operatorname{BR}\left(B^{-} \rightarrow \pi^{0} K^{-}\right)$, the $\bar{b} \rightarrow \bar{s}$ electroweak penguin amplitude $\left(c_{u}-c_{d}\right) P_{\mathrm{EW}}^{\prime}$ 
can be determined (see Fig. 1 given in [22]). Note that all involved branching ratios are expected to be at the $\mathcal{O}\left(10^{-5}\right)$ level [28] and that no time-dependent measurements are needed.

- In the $2^{\text {nd }}$ - or probably $3^{\text {rd }}$ - generation of experiments the CKM-angle $\gamma$ can hopefully be determined by using the absolutely clean methods of [3]- [5]. The knowledge of this angle would improve the determination of the electroweak penguin amplitude $\left(c_{u}-c_{d}\right) P_{\mathrm{EW}}^{\prime}$ considerably as has been stressed in [22]. In these experiments it will hopefully also be possible to implement the approaches 24, 27] to determine the CKM-angle $\alpha$ in a clean way. The uncertainties of both methods, electroweak penguins and $S U(3)$-breaking in [24] and [27], respectively, are expected to be of the same order, i.e. $\mathcal{O}\left(\bar{\lambda}^{2}\right)$, and should be rather small. In the case of the $\alpha$-determination by means of $B \rightarrow \pi \pi$ decays [24], the electroweak penguin corrections can even be controlled in a quantitative way as has been shown in 22]. Besides the extraction of $\alpha$, the approach presented in 27] would also allow an independent determination of $\gamma$ and $z, \bar{z}$ as we have shown above. This would yield an interesting cross-check.

Let us now discuss some further applications of the approach to extract the electroweak penguin contributions to nonleptonic $B$-decays presented in [22]. They can be divided into two categories:

i) quantitative insights into the physics of the electroweak penguin operators

ii) consistency checks.

In order to work out point i), we shall use a low energy effective Hamiltonian describing $|\Delta B|=1, \Delta C=\Delta U=0$ transitions. In the case of $b \rightarrow s$ decays, the $\Delta B=-1$ part can be written in the form 29]

$$
\begin{aligned}
& \mathcal{H}_{\mathrm{eff}}(\Delta B=-1)= \\
& \quad \frac{G_{\mathrm{F}}}{\sqrt{2}}\left[V_{u s}^{*} V_{u b} \sum_{k=1}^{2} Q_{k}^{u} C_{k}(\mu)+V_{c s}^{*} V_{c b} \sum_{k=1}^{2} Q_{k}^{c} C_{k}(\mu)-V_{t s}^{*} V_{t b} \sum_{k=3}^{10} Q_{k} C_{k}(\mu)\right],
\end{aligned}
$$

where $Q_{k}$ are local four-quark operators and $C_{k}(\mu)$ denote so-called Wilson coefficient functions that have been calculated by Buras et al. 29 in renormalization group improved perturbation theory including both leading and next-to-leading order QCD corrections and leading order corrections in the QED coupling $\alpha_{\mathrm{QED}}$. As usual, $\mu$ denotes a renormalization scale $\mathcal{O}\left(m_{b}\right)$. For details concerning phenomenological applications of the next-to-leading order Hamiltonian (8) to nonleptonic $B$-decays, the reader is referred to refs. [13, 14, 30, 31] where also the exact definitions of the current-current 
operators $Q_{1 / 2}^{u}, Q_{1 / 2}^{c}$, the QCD penguin operators $Q_{3}, \ldots, Q_{6}$ and the electroweak penguin operators $Q_{7}, \ldots, Q_{10}$ can be found.

The aim of the following discussion is to derive a transparent expression that allows a quantitative test whether the electroweak penguin amplitude $\left(c_{u}-c_{d}\right) P_{\mathrm{EW}}^{\prime}$ determined by following the approach [22] is consistent with the description through the Standard Model of electroweak interactions [25]. To this end let us neglect the QCD corrections to the electroweak penguin operators. In view of the approximations that will be made in a moment, this approximation seems to be reasonable. Moreover, these QCD corrections should be small if one defines the top-quark mass properly as $\overline{m_{t}}\left(m_{t}\right)$ [32 (see also ref. [15]). Within this approximation, the Wilson coefficients of the electroweak penguin operators are given by the functions $\bar{C}_{k}^{(0)}(\mu)$ specified in refs. [13, 14. Since $\bar{C}_{8}^{(0)}(\mu)$ and $\bar{C}_{10}^{(0)}(\mu)$ vanish, we have to consider only the hadronic matrix elements of the electroweak penguin operators $Q_{7}$ and $Q_{9}$. Note that the operator $Q_{9}$ plays the most important role because of its large Wilson coefficient 13.

The modes that are used in [22] to determine the $\bar{b} \rightarrow \bar{s}$ electroweak penguins are $B^{+} \rightarrow \pi^{0} K^{+}$and $B^{-} \rightarrow \pi^{0} K^{-}$. The corresponding $\Delta B=-1$ hadronic matrix elements of the electroweak penguin operators $Q_{7}$ and $Q_{9}$ are given by

$$
\begin{aligned}
\left\langle\pi^{0} K^{-}\left|Q_{7}\right| B^{-}\right\rangle & =\left\langle\pi^{0} K^{-}\left|\left\{(\bar{u} u)_{\mathrm{V}+\mathrm{A}}-\frac{1}{2}(\bar{d} d)_{\mathrm{V}+\mathrm{A}}\right\}(\bar{s} b)_{\mathrm{V}-\mathrm{A}}\right| B^{-}\right\rangle \\
\left\langle\pi^{0} K^{-}\left|Q_{9}\right| B^{-}\right\rangle & =\left\langle\pi^{0} K^{-}\left|\left\{(\bar{u} u)_{\mathrm{V}-\mathrm{A}}-\frac{1}{2}(\bar{d} d)_{\mathrm{V}-\mathrm{A}}\right\}(\bar{s} b)_{\mathrm{V}-\mathrm{A}}\right| B^{-}\right\rangle .
\end{aligned}
$$

In the notation of Gronau et al. [20], the matrix elements (9) and (10) are incorporated in the electroweak penguin amplitude $\left(c_{u}-c_{d}\right) \bar{P}_{\mathrm{EW}}^{\prime}$. On the other hand, the colourallowed amplitude $\bar{T}^{\prime}$ is related to hadronic matrix elements of the current-current operators $Q_{1}^{u}$ and $Q_{2}^{u}$. In order to calculate this quantity, we consider the mode $\bar{B}_{d}^{0} \rightarrow$ $\pi^{+} K^{-}$since - in contrast to $B^{-} \rightarrow \pi^{0} K^{-}-$no colour-suppressed amplitude $\bar{C}^{\prime}$ is present in this case. The corresponding hadronic matrix elements of the current-current operators $Q_{1}^{u}$ and $Q_{2}^{u}$ are given by

$$
\begin{aligned}
\left\langle\pi^{+} K^{-}\left|Q_{1}^{u}\right| \bar{B}_{d}^{0}\right\rangle & =\left\langle\pi^{+} K^{-}\left|\left(\bar{s}_{\alpha} u_{\beta}\right)_{\mathrm{V}-\mathrm{A}}\left(\bar{u}_{\beta} b_{\alpha}\right)_{\mathrm{V}-\mathrm{A}}\right| \bar{B}_{d}^{0}\right\rangle \\
\left\langle\pi^{+} K^{-}\left|Q_{2}^{u}\right| \bar{B}_{d}^{0}\right\rangle & =\left\langle\pi^{+} K^{-}\left|(\bar{s} u)_{\mathrm{V}-\mathrm{A}}(\bar{u} b)_{\mathrm{V}-\mathrm{A}}\right| \bar{B}_{d}^{0}\right\rangle
\end{aligned}
$$

where $\alpha$ and $\beta$ denote $S U(3)_{\mathrm{C}}$ colour indices. Note that QCD penguin matrix elements of the current-current operators $Q_{2}^{u}$ and $Q_{2}^{c}$ [30, where the up- and charm-quarks run as virtual particles in the loops, respectively, contribute by definition to the QCD penguin amplitude $\bar{P}^{\prime}$ and not to $\bar{T}^{\prime}$. A similar comment applies also to the effects of inelastic final state interactions [33] that originate e.g. from the rescattering process $\bar{B}_{d}^{0} \rightarrow\left\{D_{s}^{-} D^{+}\right\} \rightarrow \pi^{+} K^{-}$. In our notation, these contributions are related to penguinlike matrix elements of the current-current operators and are also included in $\bar{P}^{\prime}$. 
If we introduce non-perturbative $B$-parameters and apply the $S U(3)$ flavour symmetry of strong interactions, i.e. do not distinguish between $u, d$ and $s$ quark-flavours, we obtain

$$
\begin{aligned}
\left\langle\pi^{0} K^{-}\left|Q_{7}(\mu)\right| B^{-}\right\rangle & =\frac{1}{\sqrt{2}} \frac{3}{2} \tilde{B}_{2}(\mu) f, & \left\langle\pi^{0} K^{-}\left|Q_{9}(\mu)\right| B^{-}\right\rangle & =\frac{1}{\sqrt{2}} \frac{3}{2} B_{2}(\mu) f, \\
\left\langle\pi^{+} K^{-}\left|Q_{1}^{u}(\mu)\right| \bar{B}_{d}^{0}\right\rangle & =\frac{1}{3} B_{1}(\mu) f, & \left\langle\pi^{+} K^{-}\left|Q_{2}^{u}(\mu)\right| \bar{B}_{d}^{0}\right\rangle & =B_{2}(\mu) f .
\end{aligned}
$$

In these equations, the quantity $f$ corresponds to the "factorized" matrix element $\left\langle K^{-}\left|(\bar{s} u)_{\mathrm{V}-\mathrm{A}}\right| 0\right\rangle\left\langle\pi^{+}\left|(\bar{u} b)_{\mathrm{V}-\mathrm{A}}\right| \bar{B}_{d}^{0}\right\rangle$. It is quite natural to assume $\tilde{B}_{2}(\mu) \approx-B_{2}(\mu)$, since the $\pi^{0}$-meson is a pseudoscalar particle and therefore emerges from the axial-vector parts of the quark-currents $\left[(\bar{u} u)_{\mathrm{V} \pm \mathrm{A}}-(\bar{d} d)_{\mathrm{V} \pm \mathrm{A}}\right]$ arising in the electroweak penguin operators $Q_{7}$ and $Q_{9}$. For a similar reason, the one-loop QED penguin matrix elements of the current-current operators $Q_{1 / 2}^{u}$ and $Q_{1 / 2}^{c}$ vanish and do not contribute to the amplitude $\left(c_{u}-c_{d}\right) \bar{P}_{\mathrm{EW}}^{\prime}$. The point is that the virtual photons appearing in the QED penguin diagrams generate $(\bar{u} u)_{\mathrm{V}}$ and $(\bar{d} d)_{\mathrm{V}}$ vector-currents that cannot create the pseudoscalar $\pi^{0}$-meson [15].

Combining all these considerations, we eventually arrive at

$$
\begin{gathered}
\left(c_{u}-c_{d}\right) \bar{P}_{\mathrm{EW}}^{\prime}=\frac{G_{\mathrm{F}}}{\sqrt{2}} V_{t s}^{*} V_{t b} \frac{3}{2}\left[\bar{C}_{9}^{(0)}(\mu)-\bar{C}_{7}^{(0)}(\mu)\right] B_{2}(\mu) f \\
\bar{T}^{\prime}=-\frac{G_{\mathrm{F}}}{\sqrt{2}} V_{u s}^{*} V_{u b}\left[\frac{1}{3} \frac{B_{1}(\mu)}{B_{2}(\mu)} C_{1}(\mu)+C_{2}(\mu)\right] B_{2}(\mu) f .
\end{gathered}
$$

Factorizable $S U(3)$-breaking affecting (14) can be taken into account approximately by multiplying its r.h.s. by

$$
r_{S U(3)} \equiv \frac{f_{\pi}}{f_{K}} \frac{F_{B K}\left(0 ; 0^{+}\right)}{F_{B \pi}\left(0 ; 0^{+}\right)} .
$$

Unfortunately, this correction factor depends not only on pseudoscalar meson decay constants as (5), but also on model-dependent form factors $F_{B K}\left(0 ; 0^{+}\right), F_{B \pi}\left(0 ; 0^{+}\right)$ parametrizing hadronic quark-current matrix elements [34]. The model of Bauer, Stech and Wirbel [35] yields $r_{S U(3)} \approx 1$ indicating that factorizable $S U(3)$-breaking to (14) is small. At present non-factorizable $S U(3)$-breaking is completely unknown. Consequently, we cannot include these corrections and obtain

$$
\mathcal{R}_{\mathrm{EW}} \equiv \frac{\left|\left(c_{u}-c_{d}\right) P_{\mathrm{EW}}^{\prime}\right|}{\left|T^{\prime}\right|} \approx \frac{3}{2}\left|\frac{V_{t s}^{*} V_{t b}}{V_{u s}^{*} V_{u b}}\right| \cdot\left|\frac{\bar{C}_{9}^{(0)}(\mu)-\bar{C}_{7}^{(0)}(\mu)}{\frac{1}{3} \frac{B_{1}(\mu)}{B_{2}(\mu)} C_{1}(\mu)+C_{2}(\mu)}\right| r_{S U(3)} .
$$

Applying the Wolfenstein expansion of the CKM-matrix [26] yields

$$
\left|\frac{V_{t s}^{*} V_{t b}}{V_{u s}^{*} V_{u b}}\right|=\frac{1}{\lambda^{2} R_{b}}\left(1+\mathcal{O}\left(\lambda^{2}\right)\right) \approx \frac{1}{\lambda^{2} R_{b}}
$$


where the parameter $R_{b} \equiv\left|V_{u b}\right| /\left(\lambda\left|V_{c b}\right|\right)$ is constrained by present experimental data to lie within the range $R_{b}=0.36 \pm 0.08$ [36, 37]. In order to eliminate the combination of the Wilson coefficients $C_{1 / 2}(\mu)$ and the non-perturbative $B$-parameters $B_{1 / 2}(\mu)$ appearing in the denominator of eq. (17), we identify it with the phenomenological parameter $a_{1}[35,38,39,40]$. Present experimental data implies $a_{1} \approx 1.05 \pm 0.10$. Applying the analytical expressions for the Wilson coefficients $\bar{C}_{9}^{(0)}(\mu)$ and $\bar{C}_{7}^{(0)}(\mu)$ given in refs. [13, 14], the $\mu$-dependences of these coefficients cancel explicitly and we get the $\mu$-independent result

$$
\mathcal{R}_{\mathrm{EW}} \approx \frac{\alpha_{\mathrm{QED}}}{2 \pi \lambda^{2} R_{b} a_{1} \sin ^{2} \Theta_{\mathrm{W}}}\left|5 B\left(x_{t}\right)-2 C\left(x_{t}\right)\right| r_{S U(3)},
$$

where $x_{t} \equiv m_{t}^{2} / M_{W}^{2}$ introduces a top-quark mass dependence into this expression and $B\left(x_{t}\right)$ and $C\left(x_{t}\right)$ are two of the well-known Inami-Lim functions 411. In Fig. 2 we have shown the expected dependence of $\mathcal{R}_{\mathrm{EW}}$ described by eq. (19) on the top-quark mass $m_{t}$ for various values of the CKM-parameter $R_{b}$. In drawing this figure, we have used $a_{1}=1$ and $r_{S U(3)}=1$. Note that the results for $\mathcal{R}_{\mathrm{EW}}$ shown in Fig. 2 are remarkably consistent with the naïve hierarchy given by Gronau et al. in [12, 20] yielding $\mathcal{R}_{\mathrm{EW}}=\mathcal{O}(1)$.

As a by-product, applying the results of ref. [22], we can easily estimate an upper bound for the uncertainty $\Delta \alpha$ originating from electroweak penguins in the $\alpha$ determination by means of the mixing-induced CP asymmetry $\mathcal{A}_{\mathrm{CP}}^{\text {mix-ind }}\left(B_{d} \rightarrow \pi^{+} \pi^{-}\right)$ and isospin relations among $B(\bar{B}) \rightarrow \pi \pi$ decay amplitudes [22, 24]:

$$
|\Delta \alpha| \lesssim \frac{\alpha_{\mathrm{QED}}}{2 \pi a_{1} \sin ^{2} \Theta_{\mathrm{W}}}\left|5 B\left(x_{t}\right)-2 C\left(x_{t}\right)\right| \cdot\left|\frac{V_{t d}}{V_{u b}}\right| \sin \alpha \mid .
$$

Note that the $S U(3)$-breaking factor $r_{S U(3)}$ cancels in this expression. Taking into account $\left|V_{t d}\right| /\left|V_{u b}\right| \leq 5.8$ [36, 37] and $|\sin \alpha| \leq 1$, we obtain from Fig. $2|\Delta \alpha| \lesssim 6^{\circ}$.

Let us now finally come to point ii) listed above discussing some interesting consistency checks:

- The transitions $B_{s} \rightarrow \pi^{0}(\eta, \Phi)$ :

The ratio $\mathcal{R}_{\mathrm{EW}}$ determined by following the approach [22] can be used to extract the parameter $x$ that has been introduced in [15] to describe the decay $B_{s} \rightarrow \pi^{0} \Phi$ through $x \approx-a_{1} \mathcal{R}_{\mathrm{EW}} /\left(a_{2} r_{S U(3)}\right)$. Here, $a_{2}$ is the so-called phenomenological colour-suppression factor [35, 38, 39, 40]. A detailed phenomenological analysis of the decay $B_{s} \rightarrow \pi^{0} \Phi$ has been performed in [15]. The dominance of the electroweak penguins arising in this mode, which has first been pointed out there, has been confirmed independently by the authors of refs. [17, 18]. Note that the structure of the decay $B_{s} \rightarrow \pi^{0} \Phi$ is very similar to that of the transition $B_{s} \rightarrow \pi^{0} \eta$ 
describing one diagonal of the quadrangle constructed in [20]. Unfortunately, the branching ratios for the decays $B_{s} \rightarrow \pi^{0}(\eta, \Phi)$ are very small and are expected to be of $\mathcal{O}\left(10^{-7}\right)$. Following the strategy presented above, it should be possible to predict them and the corresponding $\mathrm{CP}$ asymmetries on a rather solid ground before one can measure these quantities. It should also be possible to predict the shape of the quadrangle derived in 20].

- The transition $B_{s} \rightarrow K^{+} K^{-}$:

As has been pointed out in [22], the mixing-induced CP-violating asymmetry $\mathcal{A}_{\mathrm{CP}}^{\text {mix-ind }}\left(B_{s} \rightarrow K^{+} K^{-}\right)$may be used to determine the amplitude $z \equiv T^{\prime} /\left|P^{\prime}\right|$ provided the CKM-angle $\gamma$ is known. On the other hand, if one follows the approach to extract these quantities presented above, the quantity $\xi_{K^{+} K^{-}}^{(s)}$ containing essentially all the information needed to describe the CP-violating effects arising in $B_{s} \rightarrow K^{+} K^{-}$(see ref. 22]) can be predicted and allows an interesting test. Although the branching ratio $\mathrm{BR}\left(B_{s} \rightarrow K^{+} K^{-}\right)$is rather promising and expected to be of $\mathcal{O}\left(10^{-5}\right)$, the large $B_{s}^{0}-\bar{B}_{s}^{0}$-mixing parameter may cause experimental problems.

- The transition $B_{d} \rightarrow \pi^{0} K_{\mathrm{S}}$ :

In the notation of Gronau et al. [20], the transition amplitude of the decay $B_{d}^{0} \rightarrow$ $\pi^{0} K^{0}$ takes the form

$$
\begin{gathered}
A\left(B_{d}^{0} \rightarrow \pi^{0} K^{0}\right)=-\frac{1}{\sqrt{2}}\left[C^{\prime}-P^{\prime}+\left(c_{u}-c_{d}\right) P_{\mathrm{EW}}^{\prime}-c_{d} P_{\mathrm{EW}}^{\prime \mathrm{C}}\right] \approx \\
\frac{1}{\sqrt{2}}\left[P^{\prime}-\left(c_{u}-c_{d}\right) P_{\mathrm{EW}}^{\prime}\right] \approx A\left(\bar{B}_{d}^{0} \rightarrow \pi^{0} \bar{K}^{0}\right),
\end{gathered}
$$

where we have performed the same approximations as in [22], i.e. have neglected the colour-suppressed tree-level and electroweak penguin contributions which are both $\mathcal{O}\left(\bar{\lambda}^{2}\right)$. Within this approximation, mixing-induced and direct CP violation in $B_{d} \rightarrow \pi^{0} K_{\mathrm{S}}$ (the final state $\left|\pi^{0} K_{\mathrm{S}}\right\rangle$ is an eigenstate of the $\mathcal{C P}$ operator) are characterized by

$$
\begin{aligned}
\mathcal{A}_{\mathrm{CP}}^{\text {mix-ind }}\left(B_{d} \rightarrow \pi^{0} K_{\mathrm{S}}\right) & =-\sin 2 \beta \\
\mathcal{A}_{\mathrm{CP}}^{\mathrm{dir}}\left(B_{d} \rightarrow \pi^{0} K_{\mathrm{S}}\right) & =0 .
\end{aligned}
$$

An exact definition of these asymmetries can be found in [42. Sizable nonvanishing direct $\mathrm{CP}$ violation would indicate that the approximation of neglecting the $C^{\prime}, \bar{C}^{\prime}$ topologies, which has also been performed in the approach [22] to determine $\left(c_{u}-c_{d}\right) P_{\mathrm{EW}}^{\prime}$, is not very good. In this case, (22) does not allow a clean measurement of the CKM-angle $\beta$. Comparing (22) with the absolutely clean 
asymmetry $\mathcal{A}_{\mathrm{CP}}^{\text {mix-ind }}\left(B_{d} \rightarrow \psi K_{\mathrm{S}}\right)=-\sin 2 \beta$, one can also obtain information on the quality of this approximation and moreover on the amplitudes $C^{\prime}, \bar{C}^{\prime}$. Note that the branching ratio $\mathrm{BR}\left(B_{d}^{0} \rightarrow \pi^{0} K_{\mathrm{S}}\right)=\mathrm{BR}\left(B_{d}^{0} \rightarrow \pi^{0} K^{0}\right) / 2$ can also be predicted with the help of the results of ref. [22].

- The substructure of the $B(\bar{B}) \rightarrow \pi \pi$ isospin triangles:

Combining the analyses of refs. [22, 27], we are in a position to resolve the substructure of the $B \rightarrow \pi \pi$ isospin triangle as has been shown in Fig. 3. In drawing this figure, we have neglected terms of $\mathcal{O}\left(\bar{\lambda}^{3}\right)$, e.g. colour-suppressed $\bar{b} \rightarrow \bar{d}$ electroweak penguins. A similar construction can also be performed for the corresponding CP-conjugate modes. In particular the dashed triangle, which is related to $C, T$ and $C+T$, can be fixed. The theoretical accuracy of the determination of the amplitudes $C$ and $T$ is limited by the unknown $\mathcal{O}\left(\bar{\lambda}^{2}\right)$ exchange and $S U(3)$-breaking topologies $E$ and $P_{3}$, respectively [12]. Taking into account 20]

$$
A\left(B_{s}^{0} \rightarrow \pi^{0} \bar{K}^{0}\right)=-\frac{1}{\sqrt{2}}\left[C-P+\left(c_{u}-c_{d}\right) P_{\mathrm{EW}}\right],
$$

where colour-suppressed $\bar{b} \rightarrow \bar{d}$ electroweak penguins have been neglected as in Fig. 3, a prediction of the direct and mixing-induced CP-violating asymmetries arising in the mode $B_{s} \rightarrow \pi^{0} K_{\mathrm{S}}$ is possible and would allow another consistency check if it should become possible to measure these quantities in future experiments, maybe in those of the $3^{\text {rd }}$ generation.

In summary, using the $S U(3)$ flavour symmetry of strong interactions, we have presented strategies for extracting the quantities $z, \bar{z}$ and the CKM-angle $\gamma$ which are needed as the central input for the approach to control electroweak penguins in nonleptonic $B$-decays presented in ref. [22]. In particular an approximate method making use of the modes $B^{+} \rightarrow \pi^{+} K^{0}, B_{d}^{0} \rightarrow \pi^{-} K^{+}, \bar{B}_{d}^{0} \rightarrow \pi^{+} K^{-}$and $B^{+} \rightarrow \pi^{+} \pi^{0}$ should be rather promising from the experimental point of view. We have derived a transparent analytical expression allowing a quantitative test of the question whether the ratio $\mathcal{R}_{\mathrm{EW}} \equiv\left|\left(c_{u}-c_{d}\right) P_{\mathrm{EW}}^{\prime}\right| /\left|T^{\prime}\right|$ determined by following the approach [22] is in accordance with its description through the Standard Model of electroweak interactions. As a byproduct, this formula yields an upper bound $|\Delta \alpha| \lesssim 6^{\circ}$ for the uncertainty originating from electroweak penguins in the $\alpha$-determination by means of $B(\bar{B}) \rightarrow \pi \pi$ decays [22, 24]. Some interesting predictions and consistency checks involving the decays $B_{s} \rightarrow \pi^{0}(\eta, \Phi), B_{s} \rightarrow K^{+} K^{-}$and $B_{d} \rightarrow \pi^{0} K_{\mathrm{S}}$ have also been discussed. Moreover, we have pointed out that the substructure of the $B(\bar{B}) \rightarrow \pi \pi$ isospin triangles fixing e.g. the $\mathrm{CP}$-violating asymmetries arising in the mode $B_{s} \rightarrow \pi^{0} K_{\mathrm{S}}$ can be resolved in principle. The strategies and results presented in this letter in combination with 
those of refs. [22, 27] should allow valuable insights into the world of the electroweak penguins and should furthermore provide an interesting test of the Standard Model.

I would like to thank Andrzej Buras for a very enjoyable collaboration on topics related to this paper. 


\section{References}

[1] L.L. Chau and W.-Y. Keung, Phys. Rev. Lett. 53 (1984) 1802.

[2] C. Jarlskog and R. Stora, Phys. Lett. B208 (1988) 268.

[3] M. Gronau and D. Wyler, Phys. Lett. B265 (1991) 172.

[4] I. Dunietz, Phys. Lett. B270 (1991) 75.

[5] R. Aleksan, I. Dunietz and B. Kayser, Z. Phys. C54 (1992) 653.

[6] R. Aleksan, B. Kayser and D. London, National Science Foundation preprint NSF-PT-93-4, hep-ph/9312338 (1993).

[7] M. Gronau, J.L. Rosner and D. London, Phys. Rev. Lett. 73 (1994) 21.

[8] M. Gronau, O.F. Hernández, D. London and J.L. Rosner, Phys. Rev. D50 (1994) 4529

[9] O.F. Hernández, D. London, M. Gronau and J.L. Rosner, Phys. Lett. B333 (1994) 500 .

[10] O.F. Hernández and D. London, Université de Montréal preprint UdeM-LPNTH-94-198, hep-ph/9406418 (1994).

[11] D. London, Université de Montréal preprint UdeM-LPN-TH-94-199, hepph/9406412 (1994).

[12] M. Gronau, O.F. Hernández, D. London and J.L. Rosner, Technion preprint TECHNION-PH-95-10, hep-ph/9504326 (1995).

[13] R. Fleischer, Z. Phys. C62 (1994) 81.

[14] R. Fleischer, Phys. Lett. B321 (1994) 259.

[15] R. Fleischer, Phys. Lett. B332 (1994) 419.

[16] N.G. Deshpande and X.-G. He, Phys. Lett. B336 (1994) 471.

[17] N.G. Deshpande, X.-G. He and J. Trampetic, Phys. Lett. B345 (1995) 547.

[18] D. Du and M. Yang, Institute of High Energy Physics (Beijing) preprint BIHEPTH-95-8, hep-ph/9503278 (1995).

[19] N.G. Deshpande and X.-G. He, Phys. Rev. Lett. 74 (1995) 26. 
[20] M. Gronau, O.F. Hernández, D. London and J.L. Rosner, Technion preprint TECHNION-PH-95-11, hep-ph/9504327 (1995).

[21] N.G. Deshpande and X.-G. He, University of Oregon preprint OITS-576, hepph/9505369 (1995).

[22] A.J. Buras and R. Fleischer, Universität Karlsruhe preprint TTP95-29, hepph/9507303 (1995).

[23] A.J. Buras and R. Fleischer, Phys. Lett. B341 (1995) 379.

[24] M. Gronau and D. London, Phys. Rev. Lett. 65 (1990) 3381.

[25] S.L. Glashow, Nucl. Phys. 22 (1961) 579; S. Weinberg, Phys. Rev. Lett. 19 (1967) 1264; A. Salam, in "Elementary Particle Theory", edited by N. Svartholm (Almqvist and Wiksell, Stockholm, 1968).

[26] L. Wolfenstein, Phys. Rev. Lett. 51 (1983) 1945.

[27] A.J. Buras and R. Fleischer, Universität Karlsruhe preprint TTP95-30, hepph/9507460 (1995).

[28] G. Kramer and W.F. Palmer, DESY 95-131, hep-ph/9507329 (1995).

[29] A.J. Buras, M. Jamin and M.E. Lautenbacher, Nucl. Phys. B408 (1993) 209.

[30] R. Fleischer, Z. Phys. C58 (1993) 483.

[31] G. Kramer, W.F. Palmer and H. Simma, Nucl. Phys. B428 (1994) 77.

[32] G. Buchalla and A.J. Buras, Nucl. Phys. B398 (1993) 285; Nucl. Phys. B400 (1993) 225.

[33] A.N. Kamal, Int. J. Mod. Phys. A7 (1992) 3515.

[34] A.J. Buras, J.-M. Gérard and R. Rückl, Nucl. Phys. B268 (1986) 16.

[35] M. Bauer, B. Stech and M. Wirbel, Z. Phys. C29 (1985) 637 and Z. Phys. C34 (1987) 103.

[36] A.J. Buras, M.E. Lautenbacher and G. Ostermaier, Phys. Rev. D50 (1994) 3433.

[37] A. Ali and D. London, DESY 95-148, hep-ph/9508272 (1995).

[38] M. Neubert, V. Rieckert, B. Stech and Q.P. Xu, in "Heavy Flavours", edited by A.J. Buras and M. Lindner (World Scientific, Singapore, 1992). 
[39] M.S. Alam et al., CLEO Collaboration, Phys. Rev. D50 (1994) 43.

[40] A.J. Buras, Nucl. Phys. B434 (1995) 606.

[41] T. Inami and C.S. Lim, Progr. Theor. Phys. 65 (1981) 297 and Progr. Theor. Phys. 65 (1981) 1772.

[42] R. Fleischer, Phys. Lett. B341 (1994) 205.

\section{Figure Captions}

Fig. 1: A determination of the quantities $z, \bar{z}$ and the CKM-angle $\gamma$.

Fig. 2: The dependence of $\mathcal{R}_{\mathrm{EW}} \equiv\left|\left(c_{u}-c_{d}\right) P_{\mathrm{EW}}^{\prime}\right| /\left|T^{\prime}\right|$ on the top-quark mass $m_{t}$ for $a_{1}=1, r_{S U(3)}=1$ and various values of the CKM-parameter $R_{b}$.

Fig. 3: The substructure of the $B \rightarrow \pi \pi$ isospin triangle. 
Figure 1: 
Figure 2: 
Figure 3: 
This figure "fig1-1.png" is available in "png" format from: http://arxiv.org/ps/hep-ph/9509204v1 
This figure "fig1-2.png" is available in "png" format from: http://arxiv.org/ps/hep-ph/9509204v1 
This figure "fig1-3.png" is available in "png" format from: http://arxiv.org/ps/hep-ph/9509204v1 